\title{
Association of advanced age and cancer history with autoimmune disease in melanoma patients: a cross-sectional study
}

\author{
Aaron N. Holmes ${ }^{1}$, Helen Swede ${ }^{1}$, Wendy M. Feer ${ }^{1}$, Donna Comins Pike' ${ }^{1}$, Xiaoyan Wang ${ }^{1,2}$ and \\ Upendra P. Hegde ${ }^{1^{*}}$
}

\begin{abstract}
Background: Immune-related adverse events (irAEs) are a major toxicity of immune checkpoint inhibitors. Studies have reported that pre-existing autoimmunity increases the risk of irAEs, but it remains unknown which clinical factors are linked to auto-immune disorders in cancer patients. This study aimed to evaluate if the prevalence of autoimmune diseases varied by specific cancer history and advanced age.

Methods: Our cross-sectional medical record review consisted of 291,333 patients (age, $\geq 18$ years) treated between 2000 and 2018. Patients were classified into four study groups (melanoma only, non-cutaneous solid cancer only, melanoma and non-cutaneous cancer, and no cancer history). Dependent variable was the presence of $\geq 1$ autoimmune disorders based on 98 conditions using 317 ICD codes.

Results: Non-cutaneous cancer, in the absence or presence of melanoma, was associated with a higher prevalence of autoimmunity $(16.5,95 \% \mathrm{Cl} 16.1-16.9 ; 20.0,95 \% \mathrm{Cl} 18.3-21.7$, respectively) compared to the rates in patients with melanoma only and those without cancer history $(9.3,95 \% \mathrm{Cl} 8.6-10.0 ; 6.2,95 \% \mathrm{Cl} 6.1-6.3$, respectively). Among patients with metastases at initial presentation, those in the melanoma and non-cutaneous cancer group had a prevalence of $24.0 \%$ (95\% Cl 20.1-27.9) compared to $19.1 \%$ (95\% Cl 17.2-21.0) in those without metastases. Multiple logistic regression demonstrated that patients $\geq 75$ years exhibited the highest odds of autoimmunity relative to other age groups, with age $18-34$ as the referent $(\mathrm{OR}, 1.78,95 \% \mathrm{Cl} 1.67-1.89)$.
\end{abstract}

Conclusions: Among patients with melanoma, the greatest prevalence of autoimmunity occurred with advanced age and a history of non-cutaneous cancer.

Keywords: Melanoma, Autoimmunity, Immunotherapy, Aging, Immune checkpoint inhibitors

\section{Background}

Clinically effective anti-cancer immune therapy has become possible with the advent of immune checkpoint inhibitors (ICIs). Such therapies have improved outcomes in melanoma, renal cell carcinoma, non-small cell lung

\footnotetext{
*Correspondence: uhegde@uchc.edu

1 University of Connecticut Health Center, 263 Farmington Avenue, Farmington, CT 06030, USA

Full list of author information is available at the end of the article
}

cancer, and other cancers [1]. However, patients on this therapy are at risk of developing immune-related adverse events (irAEs) that at times can be severe, unpredictable, and life threatening. The fatality rates of irAEs for anti-CTLA- 4 and anti-PD- 1 therapy are 1.08 and $0.36 \%$, respectively [2].

Meta-analyses have established that pre-existing autoimmunity increases the risk of irAEs following ICI therapy $[3,4]$. Since advanced age predisposes to both melanoma and autoimmunity, there are concerns of 
enhanced autoimmunity in senior patients with melanoma and other cancers receiving ICIs [5-7]. Interestingly, meta-analyses of large clinical trials do not see differences in irAEs by age [8-10]. By contrast, retrospective studies and meta-analyses of case reports from "realworld" experiences are mixed with some reporting higher autoimmunity in senior patients [10-12]. Some significant limitations exist in past work, as clinical trials highly select patients and many real-word case reports are lowpower studies due to low sample sizes. Large retrospective studies of health centers in the community may aid in resolving this controversy.

An understanding of underlying autoimmunity by age and cancer diagnosis may help establish irAE risk in different patient populations. To this end, we examined prevalence of autoimmunity by age and cancer diagnosis from a large single-institution database. We decided to focus on melanoma as our model cancer, because melanoma is an immunogenic cancer that responds well to ICIs, and its incidence has sharply increased in recent years among senior patients.

\section{Methods Study design}

We conducted a cross-sectional study of 291,333 patients aged 18-106years old who were treated at in-patient or out-patient sites of the University of Connecticut Health Center between 2000 and 2018. Patient information was abstracted from a GE Centricity IDX database of the electronic medical record. The sample consisted of 36,219 cancer patients and a random sample of 255,114 patients without cancer history.

\section{Independent variables and covariables}

Patients were organized into four cancer-related study groups, those with: (1) primary melanoma only ( $n=6543)$, (2) non-cutaneous malignant neoplasms only $(n=27,630)$, (3) both melanoma and non-cutaneous malignant neoplasms $(n=2046)$, and (4) patients without a cancer history $(n=255,114)$. International Classifications of Diseases, Ninth and Tenth Revisions (ICD-9, ICD-10) codes were used as follows: melanoma (ICD-9172.x; ICD-10 D03.xx, C43.x) and non-cutaneous neoplasms (ICD-9140-239 aside from 172 to 173.x, 196198.x, 216.x. 232.x, 238-239.x; ICD-10 C00-D48 aside from C43-44.x, C77-C79.x, D03-04.xx, D22-23.x, D48.x). Among cancer patients, those having regional or distant metastases at diagnosis were identified by the following codes: ICD-9196-198.x; ICD-10 C77-79.x. These codes only specified the location of the metastatic tumor, not that of the primary tumor. Patients without cancer history were selected randomly and included patients with basal cell carcinoma and squamous cell carcinoma of the skin (ICD-9173.x; ICD-10 C43.x).

The electronic databases also contained vital status and demographic information such as sex, age, race, insurance type, and smoking history. The age at the time of the first cancer diagnosis or at time of visit (non-cancer patients) was used in order to categorize patients into age groups for analyses $(18-34,35-49,50-64,65-74$, and $\geq 75$ years old). Clinical conditions existing at the time of patient's first cancer diagnosis were identified, including autoimmune disorders. Current medication regimen or clinical activity of autoimmune disorder was unavailable. This investigation was approved by the University of Connecticut Health Institutional Review Board.

\section{Outcome variable (autoimmune disorders)}

Using ICD codes, we ascertained presence of autoimmune disorders identified at or before the cancer diagnosis. A list of 317 ICD codes corresponding to 98 autoimmune conditions were queried in the database (eTable 1). Nonspecific codes that may refer broadly to infectious, allergic, and multiple other causes were excluded, such as "acute pancreatitis, unspecified" (577.0, K85.0). Autoimmune conditions developed after the first recorded date of cancer diagnosis were excluded.

\section{Statistical analyses}

The chi-square test was performed to compare differences in autoimmune prevalence in the four patient groups. Multiple logistic regression was performed to characterize the relationship between autoimmune status and age groups. Relevant demographic and clinical factors were analyzed as potential confounders, which included sex, race, smoking history, cancer type, and presence of metastases. A second regression analysis was performed that considered insurance, as a proxy for age and income, instead of age group; insurance and age group were studied in separate models due to the inherent correlation between age and Medicare status. Odds ratios were calculated with $95 \%$ confidence intervals using the coefficient of the regression's best fit line. Statistical significance was set at $p \leq 0.05 ; p$-values were modified for multiple comparisons using the Bonferroni adjustment. Analyses were performed in RStudio.

\section{Results}

We studied 291,333 patients aged 18-106years old in order to ascertain potential age-related differences in autoimmunity among patients with and without cancer histories (Table 1). In all groups, there was a majority of female patients. The median age in all study groups was between 62 and 64 years old. The majority of patients 
Table 1 Characteristics of Patients by Cancer Status

\begin{tabular}{|c|c|c|c|c|}
\hline & $\begin{array}{l}\text { Melanoma } \\
\mathrm{n}(\%)^{\mathrm{a}}\end{array}$ & $\begin{array}{l}\text { Melanoma and Non- } \\
\text { Cutaneous Cancer } \\
\mathrm{n}(\%)\end{array}$ & $\begin{array}{l}\text { Non-Cutaneous Cancer } \\
\text { n (\%) }\end{array}$ & $\begin{array}{l}\text { No History of Cancer } \\
\mathrm{n}(\%)\end{array}$ \\
\hline Total & 6543 & 2046 & 27,630 & 255,114 \\
\hline \multicolumn{5}{|l|}{ Sex } \\
\hline Male & $3345(51.1)$ & $1039(50.8)$ & $11,309(40.9)$ & $108,568(42.6)$ \\
\hline Female & $3198(48.9)$ & $1007(49.2)$ & $13,736(59.0)$ & $146,536(57.4)$ \\
\hline Undetermined & $\mathrm{n} / \mathrm{a}$ & $\mathrm{n} / \mathrm{a}$ & $1(<1)$ & $10(<1)$ \\
\hline \multicolumn{5}{|l|}{ Age at Presentation, $y$} \\
\hline Median & 62 & 64 & 62 & 62 \\
\hline $18-34$ & $393(6.0)$ & $67(3.3)$ & $1552(5.6)$ & $24,415(9.6)$ \\
\hline $35-49$ & $1182(18.1)$ & $333(16.3)$ & $4083(14.8)$ & $35,915(14.1)$ \\
\hline $50-64$ & $2033(31.1)$ & $670(32.7)$ & $9529(34.5)$ & $83,749(32.8)$ \\
\hline $65-74$ & $1376(21.0)$ & $476(23.3)$ & 5981 (21.6) & $43,892(17.2)$ \\
\hline $75+$ & $1559(23.8)$ & $500(24.4)$ & 6485 (23.5) & $67,143(26.3)$ \\
\hline \multicolumn{5}{|l|}{ Race/ethnicity } \\
\hline White, non- Hispanic & $5269(80.5)$ & $1927(94.2)$ & 23,097 (83.6) & $184,554(72.3)$ \\
\hline Black, non-Hispanic & $16(<1)$ & $33(1.6)$ & $2491(9.0)$ & $17,818(6.9)$ \\
\hline Hispanic & $8(<1)$ & $2(<1)$ & $452(1.6)$ & $8422(3.3)$ \\
\hline Asian \& Pacific Islander & $8(<1)$ & $3(<1)$ & $291(1.1)$ & $3063(1.2)$ \\
\hline Other $^{c}$ & $1(<1)$ & $\mathrm{n} / \mathrm{a}$ & $30(<1)$ & $304(<1)$ \\
\hline Unknown & $1241(19.0)$ & $81(4.0)$ & $1269(4.6)$ & $40,953(16.1)$ \\
\hline \multicolumn{5}{|l|}{ Year } \\
\hline 2000-2010 & 4163 (63.6) & $1285(62.8)$ & $18,303(66.2)$ & $152,542(59.8)$ \\
\hline $2011-2018$ & $2380(36.4)$ & $761(37.2)$ & 9327 (33.8) & $102,572(40.2)$ \\
\hline \multicolumn{5}{|l|}{ Health Insurance } \\
\hline Medicare & $3370(51.5)$ & $1243(60.8)$ & $14,836(53.7)$ & $113,240(44.4)$ \\
\hline Medicaid & $135(2.1)$ & $75(3.7)$ & $2662(9.6)$ & $25,418(10.0)$ \\
\hline Private & $2964(45.3)$ & $706(34.5)$ & $9764(35.3)$ & $108,291(42.4)$ \\
\hline Self-pay & $74(1.1)$ & $22(1.1)$ & $367(1.3)$ & $8128(3.2)$ \\
\hline Other $^{d}$ & $\mathrm{n} / \mathrm{a}$ & $\mathrm{n} / \mathrm{a}$ & $1(<1)$ & $37(<1)$ \\
\hline
\end{tabular}

${ }^{a}$ Percentages refer to total size of study group

${ }^{a}$ Includes patients with basal and squamous cell skin cancer

' Native American/Alaskan Native and Multiracial

d Described as 'other,',refunds,',contract-other,' or 'contract revenue'

were first diagnosed between 2000 and 2010 and had Medicare coverage.

\section{Prevalence of autoimmune disorders}

The five most prevalent disorders among all patients were: Lichen planus (1.5\%), type 1 diabetes (1.2\%), rheumatoid arthritis (0.9\%), systemic lupus erythematosus (0.7\%), and scleroderma (0.5\%; eTable 2). In all study groups, women had a higher prevalence of autoimmunity.

Autoimmune prevalence was found to vary by study group. In the absence or presence of melanoma, a history of non-cutaneous cancers was associated with a higher prevalence of autoimmunity (Fig. 1); among those with both melanoma and non-skin cancers, the prevalence of autoimmunity was $20.0 \%$ (95\% CI $18.3-$ 21.7), and among those with non-cutaneous cancers alone, the prevalence was $16.5 \%$ (95\% CI 16.1-16.9). Both rates were approximately triple that among patients without cancer history $(6.2,95 \%$ CI 6.1-6.3, $p<0.001)$. Of note, melanoma patients without a history of non-cutaneous cancer had the lowest prevalence of autoimmunity across the cancer groups (9.3, 95\% CI 8.6-10.0). Further analyses demonstrated increased prevalence of autoimmunity among patients with benign vs malignant tumors, control patients with non-melanoma skin cancers vs those without, and melanoma patients treated before vs after 2011 (eTable 3). 


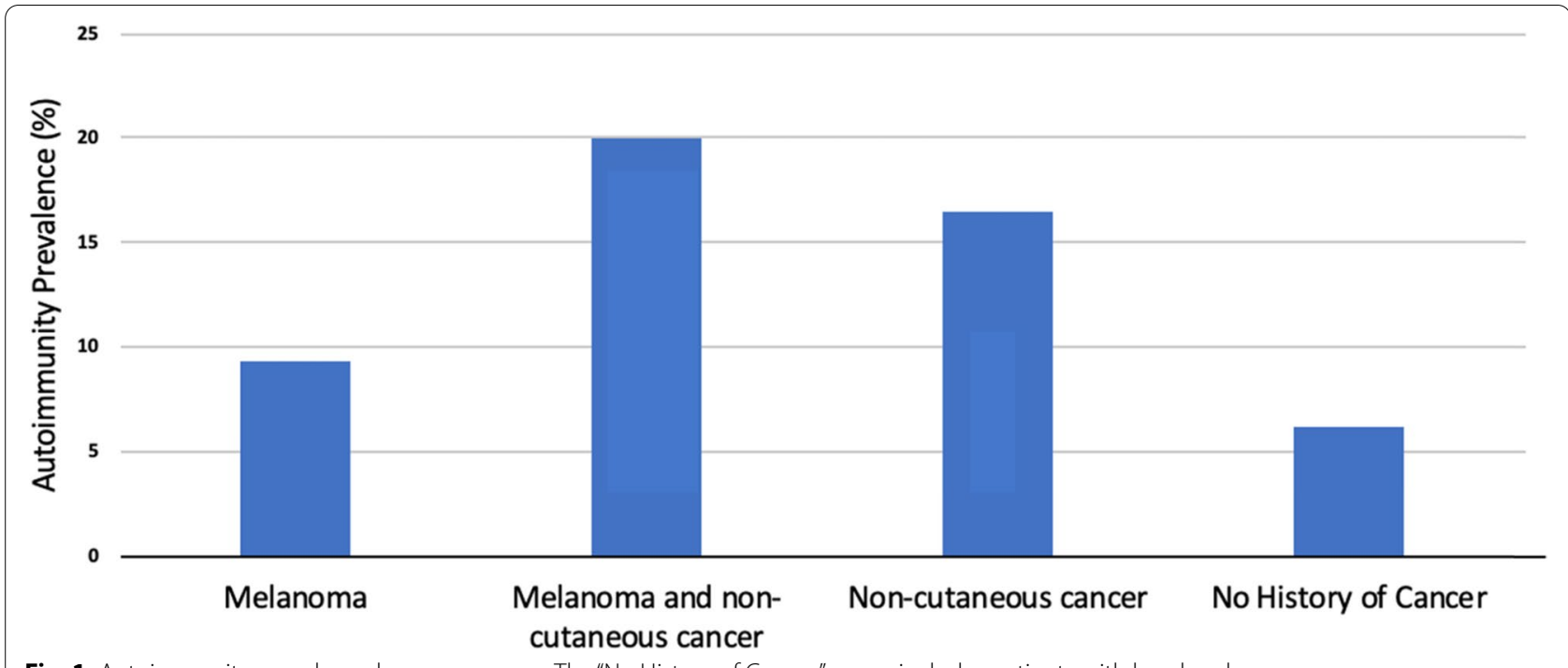

Fig. 1 Autoimmunity prevalence by cancer group. The "No History of Cancer" group includes patients with basal and squamous cancers

\section{Patients with metastatic cancer at diagnosis}

A total of 2285 patients with evidence of metastatic disease were studied (eTable 4). Patients with metastatic non-cutaneous cancer were considerably older than their group in aggregate (median 69 years vs 62 ), while metastatic melanoma patients with and without non-cutaneous cancers had comparable median ages to their group in aggregate (65 vs 64 and 60 vs 62, respectively). We show evidence of variability in metastasis prevalence by cancer group. Only $1.8 \%$ of patients with primary melanoma alone had metastases, compared to $8.3 \%$ of noncutaneous cancer patients and $24.0 \%$ of patients with both melanoma and non-cutaneous cancers (eTable 3). As seen with all other analyses, women with metastases had a higher prevalence of autoimmune conditions compared to men diagnosed with metastatic disease (e'Table 5).

Autoimmune prevalence among those with metastases appeared similar to that of their corresponding study group without metastases (Table 2), with the exception of the study group with both melanoma and non-cutaneous cancers: those with metastases had a higher autoimmune disease prevalence of $24.0 \%$ (95\% CI 20.1-27.9) compared to $19.1 \%(95 \%$ CI 17.1-21.1) in patients without metastases $(p=0.020)$.

\section{Risk factors associated with autoimmunity}

A multiple logistic regression was conducted in order to identify independent factors associated with the presence of autoimmune conditions among all patients studied. Age groups greater than age 34 had increased odds of autoimmunity relative to the 18-34 age group
Table 2 Autoimmune Prevalence by Metastatic Cancer

\begin{tabular}{llll}
\hline & $\begin{array}{l}\text { Melanoma } \\
(\boldsymbol{n}=\mathbf{1 1 6})\end{array}$ & $\begin{array}{l}\text { Melanoma and Non- } \\
\text { Cutaneous Cancer } \\
(\boldsymbol{n}=\mathbf{4 7 1 )}\end{array}$ & $\begin{array}{l}\text { Non- } \\
\text { Cutaneous } \\
\text { Cancer } \\
(\boldsymbol{n}=\mathbf{1 6 9 8})\end{array}$ \\
\hline $\begin{array}{l}\text { Autoimmune } \\
\text { Prevalence } \\
(\mathbf{\%})^{\mathbf{a}}\end{array}$ & $12(10.3)$ & $113(24.0)$ & $281(16.5)$ \\
$\begin{array}{l}\text { Age at presentation, } \mathbf{y} \\
\text { 18-34 }\end{array}$ & $1(14.3)$ & $3(25.0)$ & \\
$35-49$ & $2(9.1)$ & $20(27.0)$ & $6(22.2)$ \\
$50-64$ & $3(7.1)$ & $29(32.2)$ & $10(8.9)$ \\
$65-74$ & $2(10.5)$ & $23(20.1)$ & $64(13.1)$ \\
$75+$ & $3(12.0)$ & $38(31.4)$ & $116(21.4)$ \\
\hline
\end{tabular}

${ }^{\text {a }}$ Percentages refer to respective size of subgroup among metastatic cancer

${ }^{\mathrm{b}}$ Includes patients with basal and squamous cell skin cancer

(Table 3). Further, the 75 and older age group, compared to the 18-34 age group, was correlated with the highest odds of autoimmunity out of all age groups (Adjusted OR, 1.78, 95\% CI 1.67-1.89; $p<0.001$ ), which was found to be higher than females versus males (Adjusted OR, 1.53, 95\% CI 1.48-1.57) or a history of melanoma versus no cancer (Adjusted OR, 1.59, 95\% CI 1.46-1.73). The two greatest factors associated with autoimmunity overall were a history of non-cutaneous cancer with and without melanoma (Adjusted OR, 3.22, 95\% CI 2.87-3.61; 2.46, 95\% CI 2.37-2.56, respectively; $p<0.001)$. A calculation of the interaction contrast for this model determined that non-cutaneous cancer has an additive effect on the relationship between melanoma history and autoimmunity $(\mathrm{IC}=0.31 \pm 0.11$, 
Table 3 Multivariable Logistic Regression Analysis of Risk Factors for Autoimmunity ${ }^{a}$

\begin{tabular}{|c|c|c|c|c|}
\hline \multirow[t]{2}{*}{ Predictor } & \multicolumn{2}{|l|}{ Unadjusted } & \multicolumn{2}{|l|}{ Adjusted } \\
\hline & OR $(95 \% \mathrm{Cl})$ & $p$-value ${ }^{b}$ & OR $(95 \% \mathrm{Cl})$ & $p$-value \\
\hline \multicolumn{5}{|l|}{ Age } \\
\hline $18-34$ & 1.00 & & 1.00 & \\
\hline $35-49$ & $1.43(1.34-1.52)$ & $<0.001$ & $1.30(1.22-1.39)$ & $<0.001$ \\
\hline $50-64$ & $1.27(1.20-1.35)$ & $<0.001$ & $1.38(1.30-1.46)$ & $<0.001$ \\
\hline $65-74$ & $1.27(1.19-1.35)$ & $<0.001$ & $1.36(1.27-1.45)$ & $<0.001$ \\
\hline $75+$ & $1.63(1.48-1.57)$ & $<0.001$ & $1.78(1.67-1.89)$ & $<0.001$ \\
\hline \multicolumn{5}{|l|}{ Sex } \\
\hline Male & 1.00 & & 1.00 & \\
\hline Female & $1.53(1.48-1.57)$ & $<0.001$ & $1.53(1.48-1.57)$ & $<0.001$ \\
\hline \multicolumn{5}{|l|}{ Race $^{c}$} \\
\hline White, non-Hispanic & 1.00 & & 1.00 & \\
\hline Black, non-Hispanic & $1.16(1.10-1.22)$ & $<0.001$ & $1.22(1.16-1.29)$ & $<0.001$ \\
\hline Hispanic & $0.80(0.74-0.88)$ & $<0.001$ & $1.04(0.95-1.14)$ & 1.00 \\
\hline Asian/Pacific-Islander & $1.04(0.91-1.18)$ & 1.00 & $1.13(1.00-1.29)$ & 0.9780 \\
\hline Native American/Alaskan Native & $1.68(1.05-2.68)$ & 0.529 & $1.72(1.07-2.78)$ & 0.4642 \\
\hline Multiracial & $0.76(0.40-1.44)$ & 1.00 & $1.02(0.54-1.95)$ & 1.000 \\
\hline \multicolumn{5}{|l|}{ Smoker Status ${ }^{c}$} \\
\hline Never Smoker & 1.00 & & 1.00 & \\
\hline Current or Past Smoker & $1.07(1.03-1.11)$ & 0.015 & $1.08(1.03-1.12)$ & 0.005 \\
\hline \multicolumn{5}{|l|}{ Cancer History } \\
\hline No History of Cancer ${ }^{d}$ & 1.00 & & 1.00 & \\
\hline Melanoma Alone & $1.55(1.43-1.69)$ & $<0.001$ & $1.59(1.46-1.73)$ & $<0.001$ \\
\hline Melanoma and Non- Cutaneous Cancer & $3.81(3.41-4.25)$ & $<0.001$ & $3.22(2.87-3.61)$ & $<0.001$ \\
\hline Non-Cutaneous Cancer & $3.00(2.89-3.10)$ & $<0.001$ & $2.46(2.37-2.56)$ & $<0.001$ \\
\hline \multicolumn{5}{|l|}{ Metastases at Diagnosis } \\
\hline Metastases Absent & 1.00 & & & \\
\hline Metastases Present & $2.76(2.48-3.08)$ & $<0.001$ & $1.05(0.94-1.18)$ & 1.000 \\
\hline
\end{tabular}

eFig. 1). There is additionally a multiplicative effect of non-cutaneous cancer on the relationship between melanoma and autoimmunity as evidenced by the distinct $95 \%$ confidence intervals. Non-Hispanic blacks had a significantly higher odds of autoimmunity relative to non-Hispanic white race (Adjusted OR 1.22, 95\% CI 1.16-1.29, $p<0.001)$. Among all races, Native Americans had the highest odds of autoimmunity, but this effect was not significant after performing a $p$-value adjustment for multiple comparisons (Adjusted OR, $1.72,95 \%$ CI 1.07-2.78, $p=0.4642$ ).

A second multiple regression model that considered insurance type instead of age group showed that Medicare patients had the highest risk of autoimmunity among all insurance types relative to private insurance holders (OR, 1.40, 95\% CI 1.36-1.45; $p<0.001$; eTable 6). Medicaid status did not significantly affect odds of autoimmunity (OR, 1.02, 95\% CI 0.97-1.08; $p=0.383$ ). Both multiple regression models analyzing age and insurance type had similar Akaike information criterion (AIC) values $(146,098 ; 145,682$, respectively) and had significant correlation for the entire model ( $p<0.001$ for both). All univariate comparisons (unadjusted) were consistent with their multivariate counterparts.

\section{Discussion}

Prevalence of autoimmunity by age

Our results suggest that prevalence of autoimmunity generally increases by age, particularly age $>75$ years old, consistent with prior work and hypotheses in gerontology 
$[13,14]$. This prevalence appears to vary by tumor type in that patients with a history of non-cutaneous cancers compared to those without a history of non-cutaneous cancers had significantly higher prevalence of autoimmunity. History of having had both melanoma and noncutaneous cancer exhibited the highest association with autoimmunity compared to the other three study groups (melanoma, non-cutaneous cancer, or no cancer history)

Our findings appear to be supported by the current understanding of aging physiology. Increased tumor burden, either in metastatic disease or advanced age, are likely associated with pro-inflammatory cytokines that contribute to autoimmunity $[15,16]$. Aging is shown to promote the ability of CD4+ $\mathrm{T}$ cells to generate an IL-17 response that promote autoimmunity in humans [17]. Tumor neoantigens have been linked to immune responses that have capacity to cross react with host tissues. Scleroderma is a well-studied example wherein patients diagnosed with lung cancer can develop interstitial lung disease through unique antibodies to tumor epitopes [18]. Similarly, there are associations between occult malignancy and dermatomyositis, polymyositis, rheumatoid arthritis, and systemic lupus erythematosus $[19,20]$. It has been posited that autoimmunity may be reactive to an occult malignancy in the absence of an infectious state [21]. This theory may provide some rationalization as to why rates of autoimmunity were relatively high in patients with both benign and malignant tumors in our investigation. Additionally, autoimmune disorders can promote an inflammatory state that leads to cancer, particularly in lymphomas.

While the connection between aging and cancer is well-established, our study is the first large-scale observational study using real-world data, to our knowledge, reporting associations with autoimmunity. Given the link between autoimmunity and moderate to severe adverse events in ICI therapy, current NCCN Guidelines state that patients with neurologic and life-threatening autoimmune disease are recommended against receiving therapy [22]. Recommendations for senior patients and a full range of autoimmune disorders, however, are not included in recommendations. Further, the marked increase in autoimmunity odds observed at age 75 suggests associated changes in the immune system are seen in this population. This finding also suggest that age of 75 years also represent a critical point for collection of pertinent patient history prior to treatment with ICIs.

Future epidemiologic studies of prevalence of autoimmunity across the life span are suggested as there is a dearth of evidence to date in cancer patients. Given this lack of knowledge, it is difficult to place our prevalence data in context. For example, our overall autoimmunity prevalence of $7.3 \%$, however, is somewhat consistent NIH 2005 estimate in the U.S. (5-8\%), but higher than the worldwide estimate from Hayter's meta-analysis $(4.6 \%)[23,24]$. Our dataset notably looked at 98 conditions instead of a list of 81 conditions reported previously by Hayter. As with all EHR studies, such as ours, there are potential diagnostic inaccuracies that may impact our results. For example, urticaria secondary to an allergen can be incorrectly coded as "idiopathic" (ICD-9: 708.1, ICD-10: L50.1) when the condition should have been coded as "allergic urticaria" (ICD-9: 708.0, ICD-10: L50.0).

\section{Prevalence of autoimmunity in cancer patients}

Our data demonstrate that cancer patients have a higher prevalence of autoimmunity compared to the non-cancer patient population. Cancer patients have an increased risk of subsequent autoimmunity, such as in paraneoplastic syndromes, and patients with certain autoimmune conditions have an increased risk of cancer [25, 26]. As autoimmune diagnoses were largely coded in the database at the time of cancer diagnosis, it remains uncertain which diagnosis came first. Thus, our work cannot support either directional arrow. Interestingly, while the autoimmune disease prevalence among melanoma patients $(9.3 \%)$ was significantly higher than that of the non-cancer population (6.2\%), the prevalence was significantly lower than those with a history of non-cutaneous cancer, with or without melanoma. The data are roughly consistent with limited past epidemiological studies on cancer patients. In those with lung and renal cancer, the prevalence of autoimmunity, using a shortened list of about 40 autoimmune conditions, is estimated to be $25 \%$ $[27,28]$. In one study that examined melanoma patients and included 147 different autoimmune diagnoses, the prevalence of autoimmunity was found to be $20.5 \%$, which is close to our rate of melanoma patients with noncutaneous cancer [29].

Our study showed a significant effect of metastases in patients with both melanoma and non-cutaneous cancers. These patients had a $26 \%$ metastases-associated increase in autoimmune prevalence. Our results are similar to a study that that used a looser case definition for autoimmunity and found a $43 \%$ relative increase in autoimmune disease prevalence among melanoma patients with metastases compared to those without [29]. The disparate findings suggest that the specificity of autoimmune case definitions may impact results.

\section{Sex and race}

We found an odds ratio for autoimmunity of 1.53 for female sex vs male sex, which is slightly less than the relative risk of 2.4 calculated by Hayter et al. [26] Our data 
also show that non-Hispanic black and Native American race are associated with autoimmunity, which is consistent with a recent large national database study that found an association with these groups and multiracial individuals [30]. We did not find a significant association between Asian-American or multiracial race and autoimmunity, but their results were limited somewhat by small sampling. While the influence of sex and race on autoimmunity is incompletely understood, it is thought to be a combination of genetic and environmental factors [30].

\section{Limitations}

This study is limited by incomplete clinical information in the medical record as well as inherent generalizability concerns from a single-institutional report. For example, severity, control, and activity of autoimmune conditions were not available consistently. Similarly, prognostic factors such as frailty, Charlson Comorbidity Index, ECOG score, and specific cancer stage or grade were not included in analyses. Additionally, the database does not specify which cancer site is linked to ICD codes of metastases, so it is uncertain whether melanoma metastases impact autoimmunity in the combined melanoma and non-cutaneous group. Selection bias might be a concern given that our sample is not population-based. Regarding the link between age and autoimmunity, for example, young patients are less likely to seek routine medical attention in a hospital-based clinic and may receive fewer diagnoses of autoimmunity as a result. However, to illustrate, aged $18-34$ years old represented $9.6 \%$ of the study group without cancer compared to $3.3-6.0 \%$ in the cancer study groups.

\section{Conclusions}

Our findings highlight how non-cutaneous cancer history, non-Hispanic black race, Native American race, and age $\geq 75$ are strongly associated with underlying autoimmunity. While we did not study irAEs, the significantly increased prevalence of autoimmunity in these clinical sub-groups, particularly the older patient, suggests a higher risk for adverse events during ICI treatment as pre-existing autoimmunity has been shown to increase this likelihood [3, 4]. Structured history-taking and closer monitoring in patients $>75$ undergoing ICI therapy, therefore, may be recommended as they are increasingly becoming eligible for this therapy, and autoimmune manifestations of underlying disease may be atypical, undiagnosed, or occult in this population [13]. Future prospective studies are needed to determine causal links between autoimmunity, irAE development, and survivorship.

\section{Abbreviations}

AIC: Akaike information criterion; ICD: International Classification of Disease; ICl: Immune checkpoint inhibitor; irAE: Immune-related adverse events.

\section{Supplementary Information}

The online version contains supplementary material available at https://doi. org/10.1186/s12885-021-09001-1.

Additional file 1 : eFigure 1. Risk difference for autoimmunity between patients with melanoma alone versus those with melanoma and noncutaneous cancers. An additive interaction was identified using the odds of the fitted multiple regression model for autoimmune status that included cancer history, age, sex, race, smoking history, and presence of metastases. The interaction contrast was found to be $0.29 \pm 0.12$. Bars indicate $95 \%$ confidence interval for risk differences. eTable 1. Autoimmune Conditions Queried in Database. eTable 2. Most Common Autoimmune Conditions Studied by Cancer Status. eTable 3. Comparison of Autoimmune Prevalence Among Select Groups ${ }^{\text {a }}$. eTable 4. Summary Statistics for Patients With Metastases. eTable 5. Autoimmune Prevalence by Metastatic Cancer Type with Additional Parameters. eTable 6. Multivariate Logistic Regression Analysis of Factors Predicting Autoimmunity with Insurance Type ${ }^{a}$.

\section{Acknowledgments}

We would like to thank the UCONN School of Medicine Summer Research Fellowship (Aaron Holmes) and Jane Lublin and Richard Lublin (Upendra Hegde) for funding this project.

\section{Authors' contributions}

ANH, BA.: Conceptualization, data curation, formal analysis, funding acquisition, investigation, methodology, validation, visualization, writing-original draft and editing. HS, PhD: Methodology, supervision, validation, writing-review and editing. WMF, BA: Data curation, investigation, software, validation, and writing-review and editing. DCP, BA: Data curation, investigation, software, and writing-review and editing. XW, PhD: Methodology, supervision, and writingreview and editing. UPH, MD: Conceptualization, methodology, supervision, validation, writing-original draft and editing. All authors have read and approved this manuscript.

Funding

Funding was provided by the UCONN School of Medicine Summer Research Fellowship and the Jane Lublin and Richard Lublin Fund.

\section{Availability of data and materials}

The datasets generated and analyzed during the current study are not publicly available due to its inclusion of health information, but are available from the corresponding author on reasonable request.

\section{Declarations}

\section{Ethics}

Study was approved by the University of Connecticut Health Institutional Review Board (IRB\# 19X-234-1). Informed consent was waived by the IRB due to the retrospective nature of the data. No protected health information was collected for this project. All the experimental protocol involving human data was in accordance with UConn Health's institutional guidelines.

Consent for publication

Not applicable.

Competing interests

There are no conflicts of interest to report.

\section{Author details}

${ }^{1}$ University of Connecticut Health Center, 263 Farmington Avenue, Farmington, CT 06030, USA. ${ }^{2}$ Sema4, Mount Sinai Health System, Stamford, CT, USA. 
Received: 2 August 2021 Accepted: 1 November 2021

Published online: 06 December 2021

\section{References}

1. Topalian SL, Hodi FS, Brahmer JR, et al. Five-year survival and correlates among patients with advanced melanoma, renal cell carcinoma, or non-small cell lung cancer treated with nivolumab. JAMA Oncol. 2019;5(10):1411-20

2. Wang DY, Salem JE, Cohen JV, et al. Fatal toxic effects associated with immune checkpoint inhibitors: a systematic review and meta-analysis. JAMA Oncol. 2018;4(12):1721-8.

3. Haanen J, Ernstoff MS, Wang Y, et al. Autoimmune diseases and immunecheckpoint inhibitors for cancer therapy: review of the literature and personalized risk-based prevention strategy. Ann Oncol. 2020;31(6):724-44.

4. Boland P, Pavlick AC, Weber J, Sandigursky S. Immunotherapy to treat malignancy in patients with pre-existing autoimmunity [published online April 16, 2020]. J Immunother Cancer. https://doi.org/10.1136/ jitc-2019-000356.

5. Vadasz Z, Haj T, Kessel A, Toubi E. Age-related autoimmunity. BMC Med. 2013;11(1):94

6. Malaguarnera M, Cristaldi E, Romano G, Malaguarnera L. Autoimmunity in the elderly: implications for cancer. J Cancer Res Ther. 2012:8(4):520.

7. Ramos-Casals M, Garcia-Carrasco M, Brito MP, Lopez-Soto A, Font J. Autoimmunity and geriatrics: clinical significance of autoimmune manifestations in the elderly. Lupus. 2003;12(5):341-55.

8. Daste A, Domblides C, Gross-goupil M, et al. Immune checkpoint inhibitors and elderly people: a review. Eur J Cancer. 2017;82:155-66.

9. Johnson DB, Sullivan RJ, Menzies AM. Immune checkpoint inhibitors in challenging populations. Cancer. 2017:123(111):1904-11.

10. van Holstein Y, Kapiteijn E, Bastiaannet E, van den Bos F, Portielje J, de Glas NA. Efficacy and adverse events of immunotherapy with checkpoint inhibitors in older patients with cancer. Drugs Aging. 2019;36:927-38.

11. Sattar J, Kartolo A, Hopman WM, Lakoff JM, Baetz T. The efficacy and toxicity of immune checkpoint inhibitors in a real-world older patient population. J Geriatr Oncol. 2019;10(3):411-4.

12. Cortellini A, Buti S, Santini D, et al. Clinical outcomes of patients with advanced cancer and pre-existing autoimmune diseases treated with anti-programmed death-1 immunotherapy: a real-world transverse study. Oncologist. 2019;24(6):e327-37

13. Watad A, Bragazzi NL, Adawi M, Toubi E, Porat BS, Shoenfeld Y. Autoimmunity in the elderly: insights from basic science and clinics-a minireview. Gerontology. 2017;63(6):515-23.

14. Aprahamian T, Takemura Y, Goukassian D, Walsh K. Ageing is associated with diminished apoptotic cell clearance in vivo. J Clin Exp Immunol. 2008;152(3):448-55

15. Balkwill F, Mantovani A. Inflammation and cancer: back to Virchow? Lancet. 2001;357(9255):539-45.

16. Mantovani A, Allavena P, Sica A, Balkwill F. Cancer-related inflammation. Nature. 2008:454(7203):436-44

17. Ouyang X, Yang Z, Zhang R, Arnaboldi P, Lu G, Li Q, et al. Potentiation of Th17 cytokines in aging process contributes to the development of colitis. Cell Immunol. 2011;266(2):208-17.

18. Shah AA, Rosen LC. Cancer and scleroderma: a paraneoplastic disease with implications for malignancy screening. Curr Opin Rheumatol. 2015:27(6):563.

19. Naschitz JE, Rosner I. Musculoskeletal syndromes associated with malignancy (excluding hypertrophic osteoarthropathy). Curr Opin Rheumatotol. 2008;20(1):100-5.

20. Carsons S. The association of malignancy with rheumatic and connective tissue diseases. Semin Oncol. 1997:24(3):360-72.

21. Johnson AK. Repercussions of occult malignancy-an etiologic basis for rheumatic disease. Med Hypotheses. 2013;80(4):447-51.

22. Thompson JA, Schneider BJ, Brahmer J, et al. NCCN guidelines insights: management of immunotherapy-related toxicities, version 1.2020: featured updates to the NCCN guidelines. J Natl Compr Cancer Netw. 2020:8(3):230-41.

23. Hayter SM, Cook MC. Updated assessment of the prevalence, spectrum and case definition of autoimmune disease. Autoimmun Rev. 2012;11(1):754-65.
24. Rose NR, Serrate-Sztein SA, Gretz E, et al. Progress in Autoimmune Diseases Research, NIH Autoimmune Diseases Coordinating Committee, National Institutes of Health. 2005.

25. Valencia JC, Egbukichi N, Erwin-Cohen RA. Autoimmunity and cancer, the paradox comorbidities challenging therapy in the context of preexisting autoimmunity. J Interf Cytokine Res. 2019;39(1):72-84.

26. Zampieri S, Valente $\mathrm{M}$, Adami N, et al. Polymyositis, dermatomyositis and malignancy: a further intriguing link. Autoimmun Rev. 2010;9(6):449-53.

27. El-Refai SM, Brown JD, Black EP, Talbert JC. Immune checkpoint inhibition and the prevalence of autoimmune disorders among patients with lung and renal cancer [published online June 1, 2017]. Cancer Inform. 2017. https://doi.org/10.1177/1176935117712520.

28. Khan SA, Pruitt SL, Xuan L, Gerber DE. Prevalence of autoimmune disease among patients with lung cancer: implications for immunotherapy treatment options. JAMA Oncol. 2016;2(11):1507-8.

29. Ma Q, Shilkrut M, Zhao Z, Li M, Batty N, Barber B. Autoimmune comorbidities in patients with metastatic melanoma: a retrospective analysis of US claims data. BMC Cancer. 2018;18(1):145.

30. Roberts M, Erdei E. Comparative United States autoimmune disease rates for 2010-2016 by sex, geographic region, and race. Autoimmun Rev. 2020;19(1):102423.

\section{Publisher's Note}

Springer Nature remains neutral with regard to jurisdictional claims in published maps and institutional affiliations.
Ready to submit your research? Choose BMC and benefit from:

- fast, convenient online submission

- thorough peer review by experienced researchers in your field

- rapid publication on acceptance

- support for research data, including large and complex data types

- gold Open Access which fosters wider collaboration and increased citations

- maximum visibility for your research: over 100M website views per year

At BMC, research is always in progress.

Learn more biomedcentral.com/submissions 